\title{
Modelling the effect of contact formation on electron transfer in single- molecule device
}

\author{
Marta M. D. Ramos ${ }^{1, a,}$, Helena M. G. Correia ${ }^{1, b}$ \\ ${ }^{1}$ Centro de Física da Universidade do Minho, \\ Campus de Gualtar 4710-057 Braga, Portugal \\ Tel.: +351 253604 330; Fax: +351253604061 \\ a marta@fisica.uminho.pt (M. M. D. Ramos) \\ ${ }^{\mathrm{b}}$ hcorreia@ fisica.uminho.pt (H. M. G. Correia)
}

*Corresponding Author

\begin{abstract}
The electric properties of single-molecule devices are very sensitive to details of contact formation between the molecule and the metallic electrodes. However the factors that control the electron transfer through the molecule in these devices, corresponding to slightly different molecule-metal attachments, are not well understood. In this work, we used a self-consistent molecular dynamics method to study the effect of symmetric and asymmetric contact realizations on electron transfer between two metallic electrodes through a spatially symmetric conjugated molecule.

Our results showed that both symmetric and asymmetric electron transfer, with respect to voltage inversion, can be obtained with the same molecule in agreement with the experiments. Besides, a central factor determining the asymmetric electron transfer through a symmetric molecule, caused by the asymmetric contact realization, is the oscillation of the entire molecule between both electrodes and its distortion.
\end{abstract}


Keywords: Quantum modelling; Electron transfer; Molecular wires, Effect of contact formation.

\section{Introduction}

Electron transport in a single-molecule junction has been a very active research field over the past decade both experimentally and theoretically ${ }^{1}$. However, there are still a large number of unresolved experimental questions concerning the effects of contact realizations on electron transfer through the molecules.

Experimental investigations on a spatially symmetric organic molecule insert between two gold electrodes, using a mechanically controlled break junction technique ${ }^{2}$ to provide an electrode pair with tunable distance, revealed that more than $50 \%$ of stable current-voltage characteristics were highly symmetric with respect to the direction of current flow. Moreover, asymmetric current-voltage curves inverted with respect to the applied voltage were obtained as a result of contacts manipulation. Asymmetry due to unequal coupling of the molecule with the contact electrodes seems to be present also in conduction measurements performed with scanning tunnelling microscopy ${ }^{3}$.

The above studies revealed that not only the molecular geometry plays an important role in controlling electron transport through the molecule, as suggested by other experimental ${ }^{4-7}$ and theoretical ${ }^{8}$ studies, but also the distance between the source and drain electrodes is strongly relevant in molecular-scale electronic conduction.

As in other areas, theoretical modelling can play an important role in understanding the basic physic of contact formation and its effect on electron transfer between the electrodes in a single-molecule junction. Since the interplay between the electronic and nuclear dynamics in such systems is an important factor with significant implications on 
electron transfer through the molecule ${ }^{9}$, a self-consistent quantum chemistry molecular dynamics method is ideally suited to study the time dependent electron transfer between two gold electrodes through a spatially symmetric conjugated molecule as a function of the applied electric field. Here we consider the chemical interaction at both polymermetal interfaces for symmetric and asymmetric contact realizations.

\section{Computational method}

Electron transfer calculations have been carried out using the CHEMOS code ${ }^{10,11}$, which performs self-consistent calculations of the electronic structure of the goldmolecule-gold system, using a semi-empirical application of the Hartree-Fock theory called CNDO (Complete Neglect of Differential Overlap), and molecular geometry relaxation, using a molecular dynamics method with the atomic forces obtained directly from the electronic structure calculation at each time-step.

The CNDO parameters (Mulliken electronegativities, bonding parameters and orbital exponents of Slater type atomic orbitals) built in the CHEMOS code are from

Pople and Beveridge ${ }^{12}$ for all the atoms of the molecule and from Baetzold ${ }^{13}$, for the gold atoms of both electrodes. The basis sets used in this work are $s$, for Hydrogen, $s p$ for atoms of the second row of the Periodic Table and spd otherwise.

\section{Results and discussion}

In order to investigate the effects of symmetric and asymmetric contact formation on electron transfer in a single-molecule junction, we applied the self-consistent quantum chemistry molecular dynamics method to the spatially symmetric molecule, $[9,10-$ Bis((2'-para-mercaptophenyl)-ethinyl)-anthracene] studied in a break junction experiment ${ }^{2}$, bound to gold atoms of two planar electrodes (see figure 1). To obtain a 
symmetric contact realization, we have relaxed the cluster geometry to equilibrium, starting off with the planar molecular configuration. The value of $2.35 \mathrm{~nm}$ was obtained for the distance between the electrodes, $d$, corresponding to the symmetric coupling situation. The asymmetry was introduced in the system by increasing the contact distance (i.e. the distance between sulphur and gold atoms) on the left hand side by 0.16 nm. When uniform electric field is applied to the molecule along the direction perpendicular to the electrodes surface, the contact atoms from both electrodes are taken to the fixed.

To understand the effects due to both the electronic coupling between the electrodes and the molecule and the coupling between electronic and nuclear vibration degrees of freedom within the molecule on electron transfer between cathode and anode, we examine the time evolution of the charge of contact atoms of both electrodes as a result of applied electric field perpendicular to the electrodes surface. The results obtained for symmetric and asymmetric contact realizations, as a function of the field strength and the applied field direction, are show in figures 2 and 3.

Our first observation is that the electron transfer between both electrodes occurs before the nuclear motions within the molecule take place and the charging of the contact atoms is different for symmetric and asymmetric contact formation. Secondly, the charge of the contact atoms changes in time differently for both contact configurations. It is also clear that the strength of the applied electric field does not change the time dependent profiles.

The periodic change in the charge and position of the carbon atoms of the molecular backbone, reported elsewhere ${ }^{9}$, leads to the periodic change in the charge of the gold atoms for the symmetric coupling situation. It can be seem from figure 2 that the charge 
of both contact atoms change symmetrically with the direction of the applied electric field.

Although in the static limit, corresponding to frozen nuclear vibrations, the asymmetric behaviour of the electron transfer with respect to the direction of the applied electric field is already evident when the distance between the molecule and the lefthand electrode is slightly modified (figure 3), the asymmetric charge transfer is clear when the relaxation of the nuclear positions in the molecule takes place. Close inspection of the molecular geometry in this case revealed that the time dependent behaviour of the charge on the gold atoms manifested in figure 3 is a consequence of the vibration motion of the entire molecule between the electrodes and the molecular distortion (see figure 4).

Since the electron lifetime of about $10^{-9} \mathrm{~s}$ on the molecular bridge estimated by Galperin et al. ${ }^{1}$ is long enough for electron-phonon interaction to take effect, the electron transfer between the electrodes through the molecule measured in the experiments is likely to be some sort of time-average. Figures 5 and 6 show the changes in the charges of the molecule and contact atoms of both gold electrodes caused by the applied electric field, for symmetric and asymmetric coupling situations when molecular motion is both prevented and allowed.

Our results suggest that electron transfer between the contact atoms of both electrodes through the symmetric molecule gives rise to the charging of that molecule, regardless the type of contact formation. It can be seen from figure 5 that the average charge stored in the molecule change symmetrically with the applied electric field for the symmetric coupling situation, which leads to a symmetric electron transfer with respect to the direction of electron flow. In this case, we found that electron-phonon interaction leads to an increase in the electron transfer between the electrodes through 
the molecule. Our calculated molecular charging without adjustable parameters is in qualitative agreement with results obtained by a self-consistent model for molecular conduction (with two adjustable parameters, the position of the Fermi energy and the sulphur-gold bond length) ${ }^{14}$. However, in the asymmetric coupling situation we show that the origin of charging-induced asymmetry in spatially symmetric molecule conductor, responsible for the asymmetric current-voltage curves ${ }^{14}$, is non-trivial and involves the vibration and distortion of the molecule between the contacts, which decreases the electron transfer through the molecule. A clear crossover between enhancement and reduction of the conductance across a single-molecule break junction due to electron-phonon interaction was also revealed recently by the experiments ${ }^{15}$.

\section{Conclusions}

The results present in this paper show the effects of both the chemical interactions between the electrodes and the molecule and the electron-phonon coupling within the molecule on electron transfer across a single-molecule junction. We have investigated the effect of symmetric and asymmetric contact formation an electron transfer through a conjugated molecule with no spatial asymmetry. We show that electron-phonon interaction leads to an increase on electron transfer across the junction for the symmetric contact formation and a decrease otherwise. We also show that the origin of the observed contact-induced asymmetry on the electron transfer is due to an asymmetric charging of the molecule with respect to the direction of the applied electric field, which involves an unequal coupling of the molecule with the contact atoms of both electrodes as a result of the vibration and the distortion of the molecule between the electrodes. 
The use of a self-consistent quantum-chemistry molecular dynamics method (with no adjustable parameters) provides not only a physical interpretation of the experiments, but also information about the nuclear dynamics of these molecular systems which can not be obtained directly from the experiments and are important for the formulation of charge transport in single-molecule junctions.

\section{Acknowledgements}

This work is part of the research projects POCTI/CTM/41574/2001 and CONCREEQ/443/EEI/2005, approved by the Portuguese Foundation for Science and Technology (FCT) and supported by the European Community Fund FEDER. 


\section{List of figure captions:}

Figure 1 - Schematic diagram of gold-[9,10-Bis((2'-para-mercaptophenyl)-ethinyl)anthracene]-gold cluster model used in this work. The dashed lines represent the position of two planar electrodes for the symmetric contact formation.

Figure 2 - The time evolution of the charge stored on the contact atoms of the left-hand side (solid lines) and right-hand side (dashed lines) shown in Figure 1 for the symmetric contact formation, when electric fields of different strengths are applied perpendicular to the electrodes surface in opposite directions.

Figure 2 - The time evolution of the charge stored on the contact atoms of the left-hand side (solid lines) and right-hand side (dashed lines) shown in Figure 1 for the asymmetric contact formation, when electric fields of different strengths are applied perpendicular to the electrodes surface in opposite directions.

Figure 4 - The geometry of the molecular show in Figure 1 for three different times and an applied electric field of $5 \mathrm{MV} / \mathrm{cm}$, when the initial contact formation $(t=0)$ is symmetric (left-hand side) and asymmetric (right-hand side).

Figure 5 - Change in the charge of the molecule and the contact atoms of left-hand (Au33) and right-hand (Au34) electrodes induced by an applied field perpendicular to the electrodes surface, for the symmetric coupling situation, when the nuclear motion is forbidden (solid marks) and allowed (open marks). 
Figure 6 - Change in the charge of the molecule and the contact atoms of left-hand (Au33) and right-hand (Au34) electrodes induced by an applied field perpendicular to the electrodes surface, for the asymmetric coupling situation, when the nuclear motion is forbidden (solid marks) and allowed (open marks). 
Modelling the effect of contact formation on electron transfer in singlemolecule device

Ramos, Marta M. D. and Correia, Helena M. G:

Figure 1

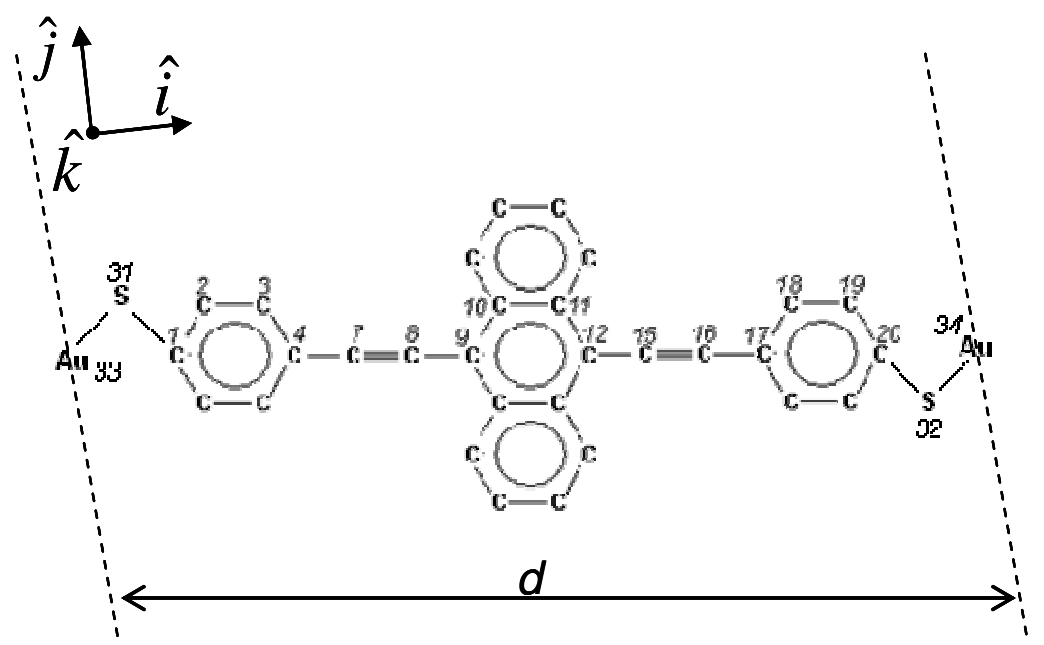


Modelling the effect of contact formation on electron transfer in singlemolecule device

Ramos, Marta M. D. and Correia, Helena M. G:

Figure 2

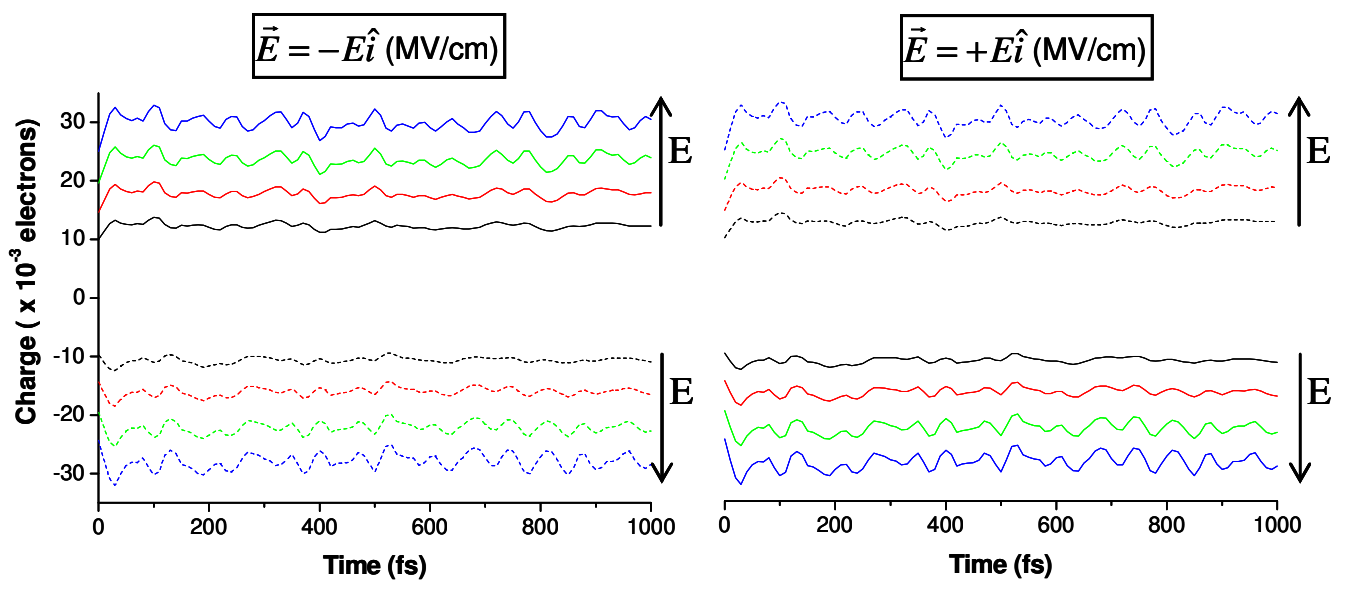


Modelling the effect of contact formation on electron transfer in singlemolecule device

Ramos, Marta M. D. and Correia, Helena M. G:

Figure 3

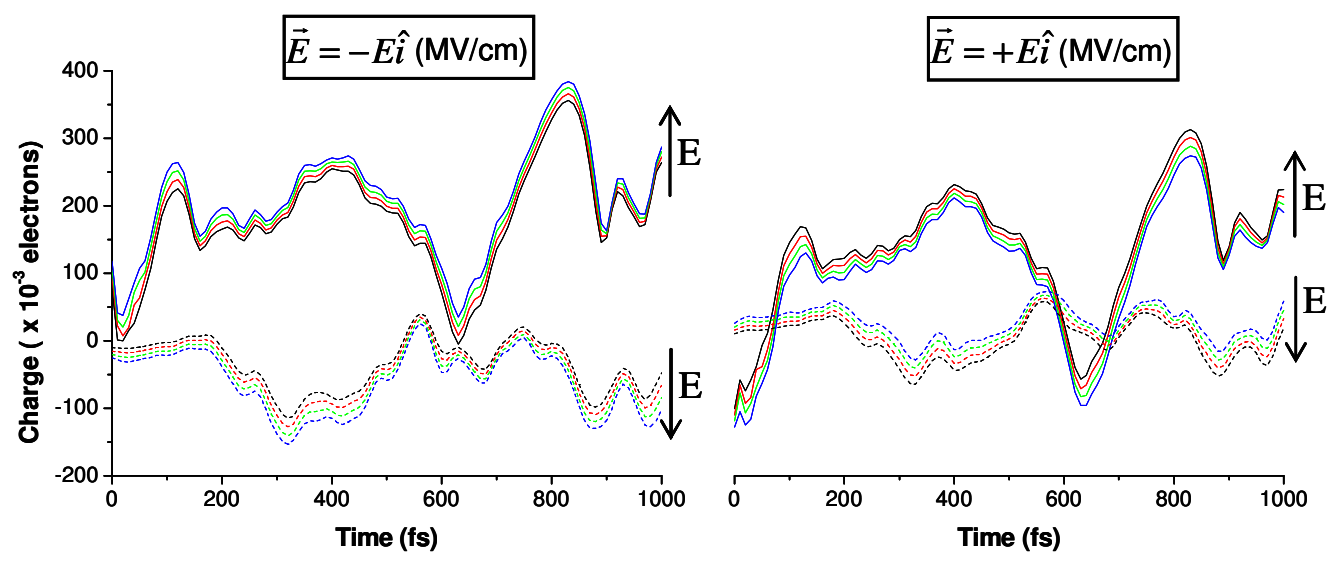


Modelling the effect of contact formation on electron transfer in singlemolecule device

Ramos, Marta M. D. and Correia, Helena M. G:

Figure 4
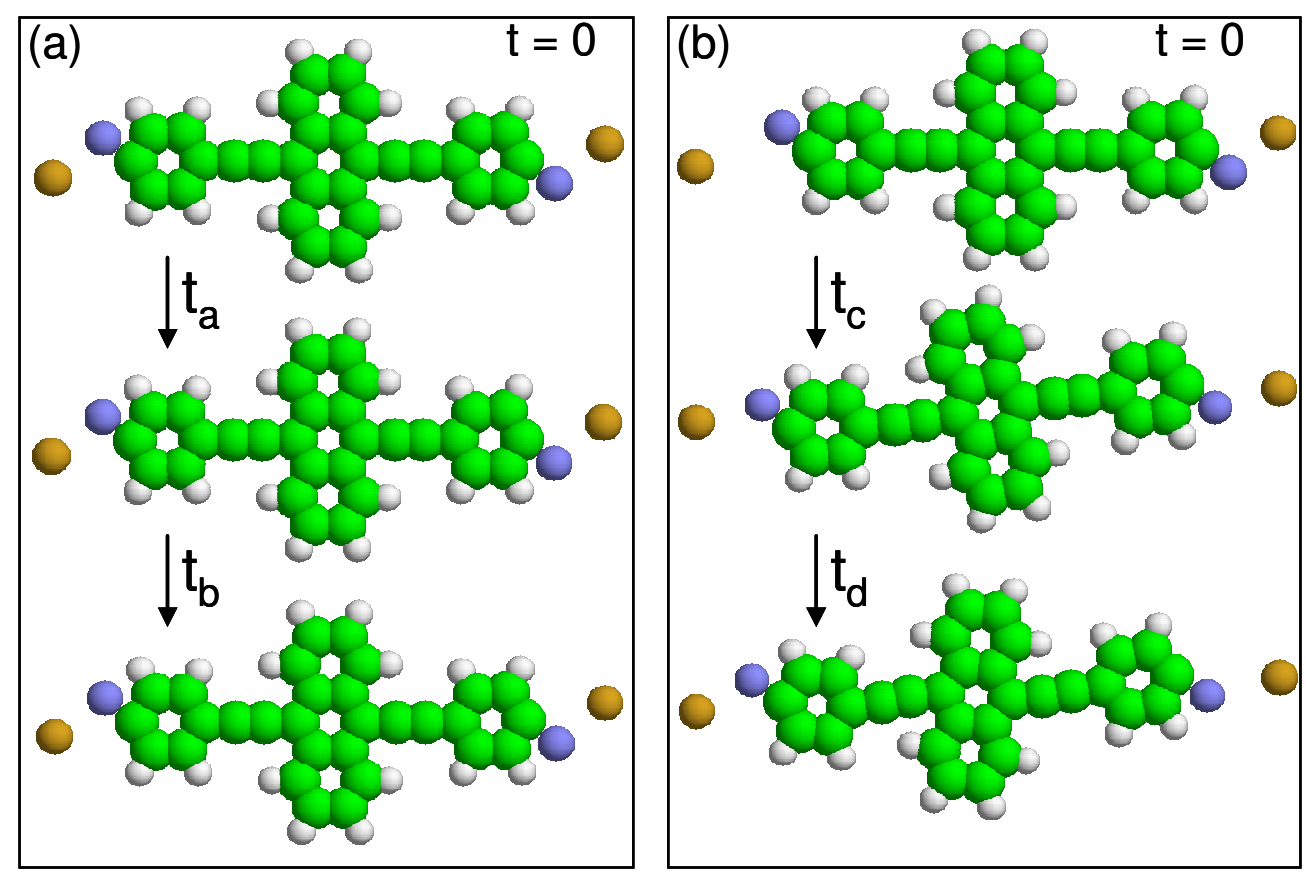
Modelling the effect of contact formation on electron transfer in singlemolecule device

Ramos, Marta M. D. and Correia, Helena M. G:

Figure 5

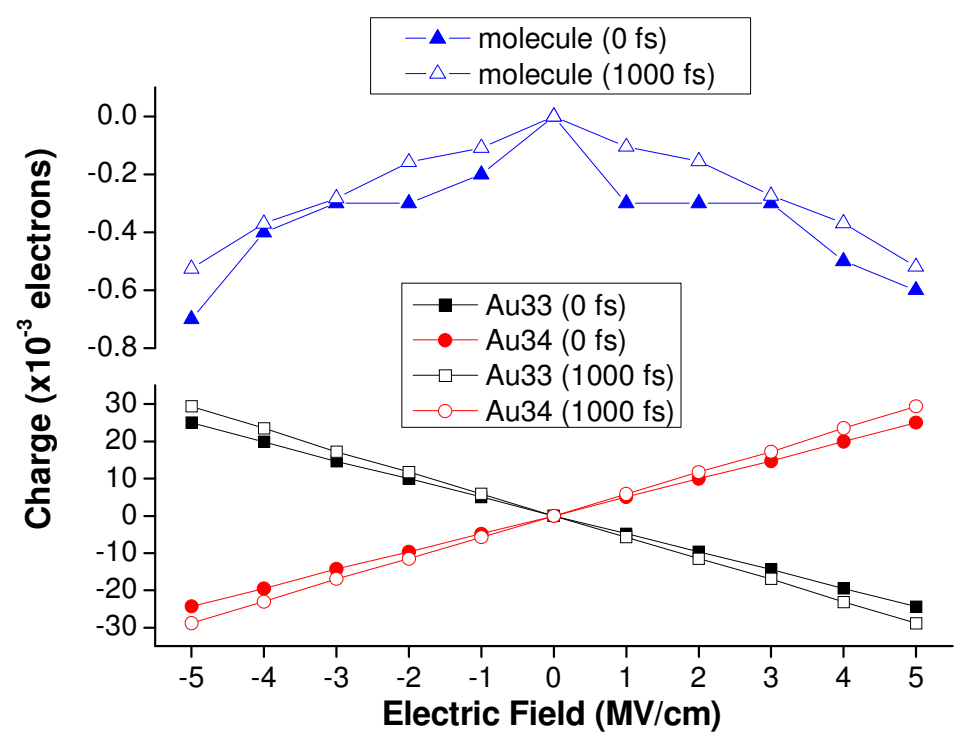


Modelling the effect of contact formation on electron transfer in singlemolecule device

Ramos, Marta M. D. and Correia, Helena M. G:

Figure 6

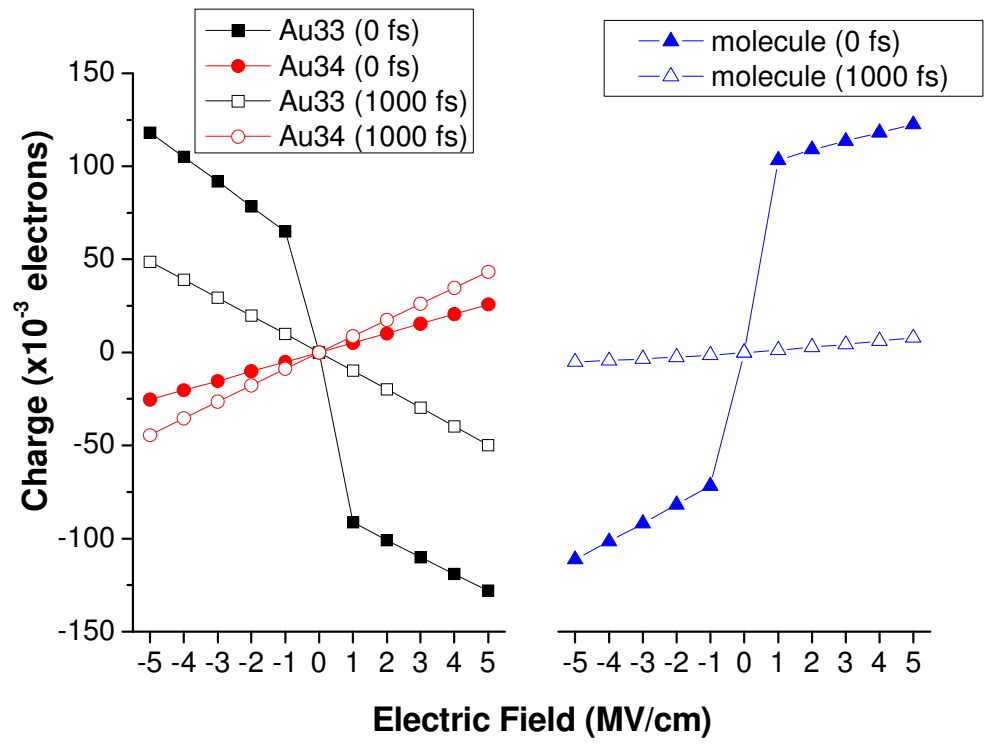




\section{References}

1. M. Galperin, M. A. Ratner and A. Nitzan, J. Phys.-Condes. Matter 19, 103201 (2007)

2. J. Reichert, R. Ochs, D. Beckmann, H. B. Weber, M. Mayor and H. von Lohneysen, Phys. Rev. Lett. 88, 176804 (2002)

3. W. D. Tian, S. Datta, S. H. Hong, R. Reifenberger, J. I. Henderson and C. P. Kubiak, J. Chem. Phys. 109, 2874 (1998)

4. M. Del Valle, R. Gutierrez, C. Tejedor and G. Cuniberti, Nat. Nanotechnol. 2, 176 (2007)

5. Z. J. Donhauser, B. A. Mantooth, K. F. Kelly, L. A. Bumm, J. D. Monnell, J. J. Stapleton, D. W. Price, A. M. Rawlett, D. L. Allara, J. M. Tour and P. S. Weiss, Science $292,2303(2001)$

6. J. Taylor, M. Brandbyge and K. Stokbro, Phys. Rev. B 68, 121101 (2003)

7. L. Venkataraman, J. E. Klare, C. Nuckolls, M. S. Hybertsen and M. L. Steigerwald, Nature 442, 904 (2006)

8. C. M. Finch, S. Sirichantaropass, S. W. Bailey, I. M. Grace, V. M. Garcia-Suarez and C. J. Lambert, J. Phys.-Condes. Matter 20, 022203 (2008)

9. H. M. G. Correia and M. M. D. Ramos, Mater. Sci. Eng. C-Biomimetic Supramol. Syst. 25, 682 (2005)

10. D. S. Wallace, D. Phil. Thesis, University of Oxford (1989).

11. D. S. Wallace, A. M. Stoneham, W. Hayes, A. J. Fisher and A. H. Harker, J. Phys.Condes. Matter 3, 3879 (1991)

12. J. A. Pople and D. L. Beveridge, Approximate Molecular Orbital Theory, McGrawHill, New York (1970).

13. R. C. Baetzold, J. Chem. Phys. 55, 4355 (1971) 
14. F. Zahid, A. W. Ghosh, M. Paulsson, E. Polizzi and S. Datta, Phys. Rev. B 70, (2004)

15. O. Tal, M. Krieger, B. Leerink and J. M. van Ruitenbeek, Phys. Rev. Lett. 100, $196804(2008)$ 